\title{
Noninvasive Temperature Estimation Based on Texture Features of Ultrasound Images for RFA
}

\section{Ming Chen}

Southeast University https://orcid.org/0000-0002-7931-0356

\section{Xingqun Zhao}

Southeast University

\section{Linfang Yao ( $\nabla$ yaolinfang47@163.com )}

Nanjing Drum Tower Hospital: Nanjing University Medical School Affiliated Nanjing Drum Tower Hospital

\section{Research}

Keywords: Ultrasound, Noninvasive temperature estimation, Radio frequency ablation, Wavelet transform, Gray-level gradient co-occurrence matrix

Posted Date: January 7th, 2021

DOl: https://doi.org/10.21203/rs.3.rs-139349/v1

License: (c) (i) This work is licensed under a Creative Commons Attribution 4.0 International License. Read Full License 


\section{$4 \quad$ Abstract}

5 Background: Hyperthermia is one of the most common therapy for tumor, in which

\section{of Ultrasound Images for RFA}

Ming Chen ${ }^{1}$, Xingqun Zhao ${ }^{1^{*}}$, Linfang $\mathrm{Yao}^{2^{*}}$ temperature monitoring is essential. Invasive temperature monitoring is mostly used in clinic at present, which leads to limited points under monitoring and extra hurt to patients. Therefore, noninvasive temperature estimation is a key and tough problem in hyperthermia. In this paper, a noninvasive temperature estimation method for hyperthermia based on B-mode ultrasonic image with wavelet transform and texture analysis is proposed.

Methods: Animal tissue both in vitro and in vivo(pig kidneys in vitro and rabbit kidney in vivo) were taken as experimental materials. Ultrasound images and temperature data were collected from radio frequency ablation experiment under approved protocol. Image subtraction and wavelet transform were performed on the ultrasound images to enhance the change during ablation, after which texture features were extracted to analyze the relationship with temperature.

Results: Texture features hybrid entropy and energy extracted from gray-level gradient co-occurrence matrix of ultrasound images processed had higher linear correlation with temperature than traditional gray value method. From in vitro and in vivo experiments, the linear correlation coefficients obtained between hybrid entropy

Corresponding authors: ${ }^{1}$ Xingqun Zhao Email address: ndt@seu.edu.cn

2 Linfang Yao Email address: yaolinfang47@163.com 
and temperature were $0.979 \pm 0.028$ and $-0.935 \pm 0.037$, respectively, and those between energy and temperature were $0.932 \pm 0.056$ and $-0.915 \pm 0.034$, respectively. In addition, the slopes of linear model obtained in vitro were relatively consistent, while those under in vivo condition had certain individual differences.

Conclusions: The results demonstrated that texture features hybrid entropy and energy of ultrasound image processed with the method proposed in this paper can be used for temperature estimation with a smaller error because of high linear correlation with temperature both in vitro and in vivo. Compared with most previous research which only based on biomaterial in vitro, the in vivo experiment conducted in this paper verified the feasibility of the noninvasive temperature estimation method proposed in practice.

Keywords: Ultrasound; Noninvasive temperature estimation; Radio frequency ablation; Wavelet transform; Gray-level gradient co-occurrence matrix

\section{Background}

Tumor hyperthermia is a therapy that raising the tumor tissue to a certain high temperature by heating to make it inactive and die. It has been widely used in clinical tumor treatment. Hyperthermia methods mainly include microwave ablation, high-energy ultrasound (HIFU), radio frequency ablation (RFA), etc. Among them, RFA has become one of the most common heating methods which using thermal effect of radio frequency current to form a high temperature thermal coagulation area to kill the tumor cells by releasing the radio frequency alternating current ${ }^{[1][2]}$. 
For tumor tissue, low $\mathrm{pH}$ environment caused by long-term energy consumption and lack of basement membrane lead to its poor heat resistance. When the temperature reaches $40{ }^{\circ} \mathrm{C} \sim 43{ }^{\circ} \mathrm{C}$, DNA damage and cell death will occur; when the temperature exceeds $45^{\circ} \mathrm{C}$ to $50^{\circ} \mathrm{C}$, the protein in normal tissue cells will be denatured and the tissue will be dehydrated ${ }^{[3]}$. Therefore, it is particularly important to make sure that the temperature is controlled within a suitable range in hyperthermia so that it can kill tumor cells effectively and avoid causing damage to surrounding normal tissues. In recent years, many noninvasive temperature estimation methods related to tumor hyperthermia have been proposed, including magnetic resonance imaging(MRI) ${ }^{[4][5]}$, electrical impedance tomography(EIT $)^{[6]}$, ultrasound, etc., among which ultrasound has become one of the most popular research directions in this field due to its various advantages of simple operation, safety, high resolution and real-time data processing. In the field of noninvasive temperature estimation based on ultrasound, the area of image-guided is always the focus of the research. In 2002, Hou studied the correlation between gray value and temperature during HIFU with pig liver in vitro, and camp up with the conclusion that gray value of the B-ultrasound image has a certain nonlinear relationship with temperature ${ }^{[7]}$. In 2010 , Yang used pig liver in vitro to study the correlation between B-ultrasound tissue texture features and temperature in microwave ablation experiment, and verified that changes in image features can reflect the temperature of tissue ${ }^{[8]}$. In 2015, Li Yong processed the B-ultrasound images obtained after HIFU of pork with method based on gray probability distribution-cross entropy, and demonstrated that the cross-entropy of the data is 
approximately linear with the temperature ${ }^{[9]}$. In 2018, Guo proposed a BEMD-RF method based on machine learning, which provides a new idea for noninvasive temperature estimation ${ }^{[10]}$. Most of the previous research conclusions are based on in vitro experiments. Therefore, in order to make the experimental results more universal, this paper took animal tissue both in vitro and in vivo as experimental materials, using wavelet transform and gray-level gradient co-occurrence matrix as image process methods to explore the linear correlation between image texture features and temperature under different conditions.

\section{Results}

After performing wavelet transform on each set of subtraction ultrasound images, the texture feature hybrid entropy and energy of the gray-level gradient co-occurrence matrix were extracted and linearly fitted with temperature data by using the least square method.

The correlation between texture features and temperature in vitro experiment are shown in Fig.1, and multiple sets of data have high consistency. The linear correlation coefficient of hybrid entropy reaches $0.979 \pm 0.028$, and the linear correlation coefficient of energy reaches $-0.935 \pm 0.037$. 


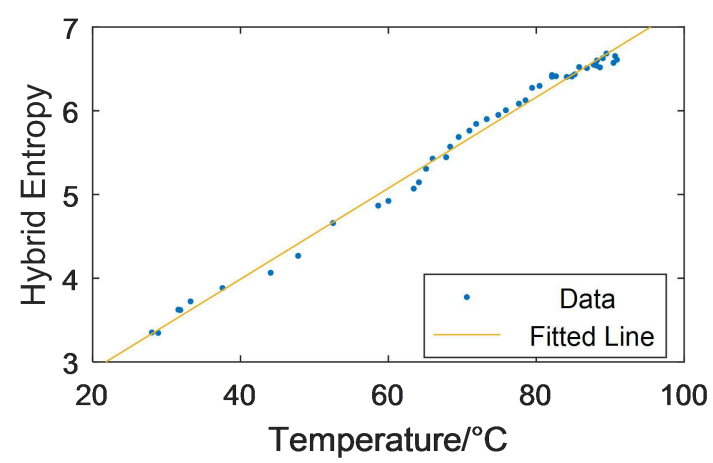

(a) Correlation between hybrid entropy and temperature

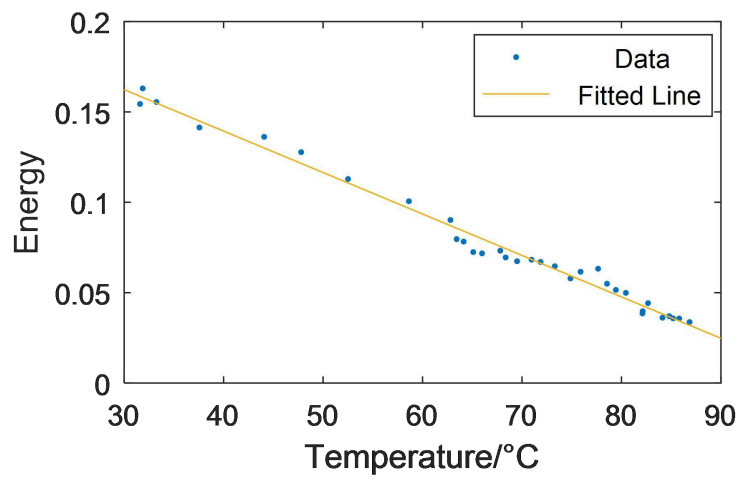

(b) Correlation between energy and temperature

Fig.1 Correlation between texture features and temperature in vitro experiment

In addition, gray value of the ultrasound image from in vitro experiment was also extracted for comparative analysis, and the correlation with temperature is shown in Fig.2.

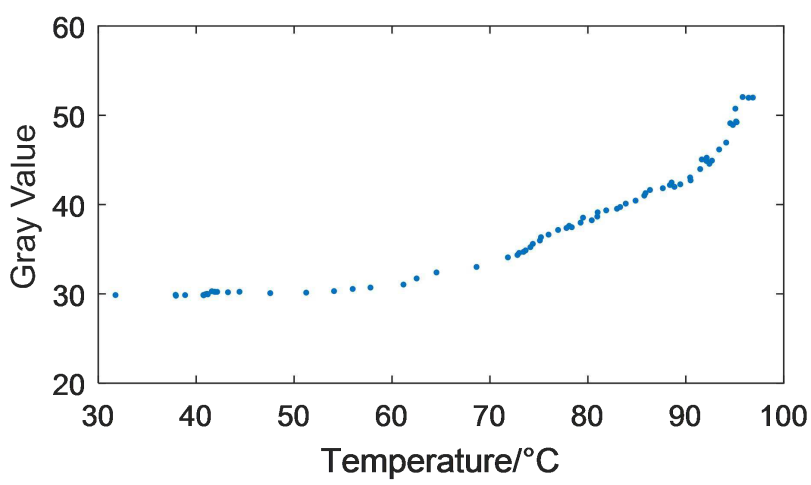

Fig.2 Correlation between gray value and temperature in vitro experiment

94 By comparing Fig.1 and Fig.2, it can be seen that under in vitro condition, gray value 
95

increases slowly in low temperature section but rapidly in high temperature section, which cannot be evaluated by a linear model and lead to a larger estimation error. However, hybrid entropy extracted from processed image increases linearly with temperature, while energy decreases linearly with temperature. In the entire temperature range, they have more obvious linear correlation with temperature. Compared with traditional gray value method, it can achieve a larger range of accurate temperature estimation.

The temperature value of the region during ablation was calculated using the correlation model of hybrid entropy and temperature obtained before, and the pseudo-color images of temperature distribution of the region at different stages of ablation are shown in Fig.3.

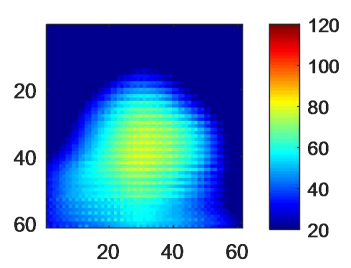

(a) Time $=100 \mathrm{~s}$

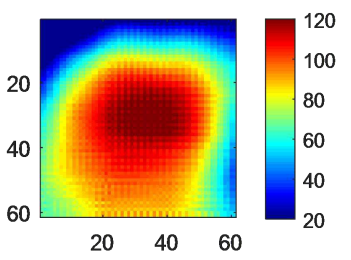

(c) Time $=300 \mathrm{~s}$

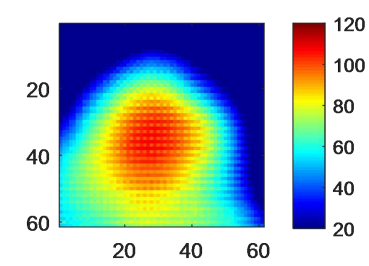

(b) Time $=200 \mathrm{~s}$

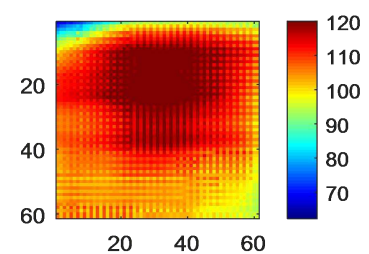

(d)Time $=400 \mathrm{~s}$

Fig .3 Pseudo-color image of temperature distribution

It can be seen from Fig. 3 that the estimated temperature decreases from the radio frequency probe to the surroundings. As the ablation heating process going on, temperature in the calculated area gradually increases, which is consistent with the 
114 actual temperature measured by the temperature probe. Therefore, it can be said that 115 the linear regression model obtained can complete the temperature estimation work in 116 the whole stage of radio frequency ablation in vitro.

117 Hybrid entropy and energy extracted from in vivo experiment based on wavelet 118 transform and the gray-level gradient co-occurrence matrix change linearly with 119 temperature, which is similar to the rule under in vitro conditions. The correlation is 120 shown in Fig.4. The linear correlation coefficient of hybrid entropy reaches $1210.932 \pm 0.056$, and the linear correlation coefficient of energy reaches $-0.915 \pm 0.034$.

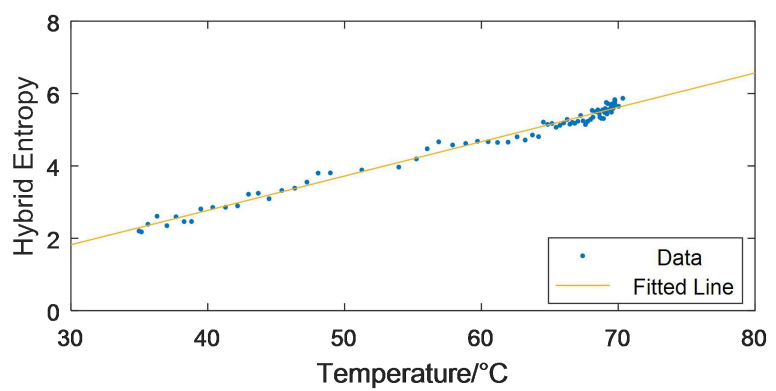

(a) Correlation between hybrid entropy and temperature

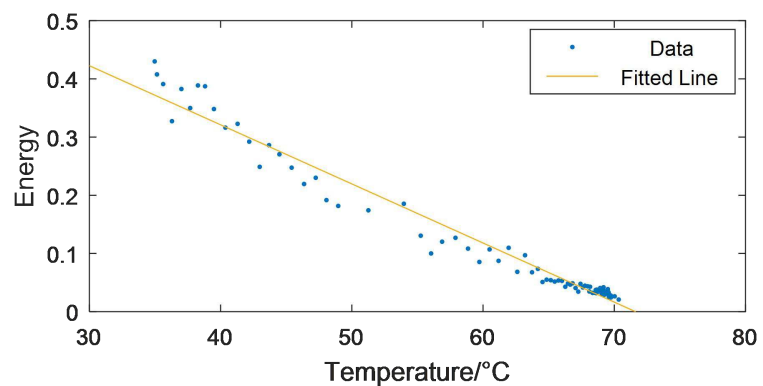

(b) Correlation between energy and temperature

Fig.4 Correlation between features and temperature from in vitro experiment

By comparing the data under two different conditions, it can be seen that the image texture features hybrid entropy and energy have a strong linear correlation with temperature both in vivo and in vitro, which verifies that the image processing method 

obtained from multiple sets of experimental data under in vitro condition have strong consistency as shown in Fig.5, while those under in vivo condition have certain individual difference as shown in Fig.6.

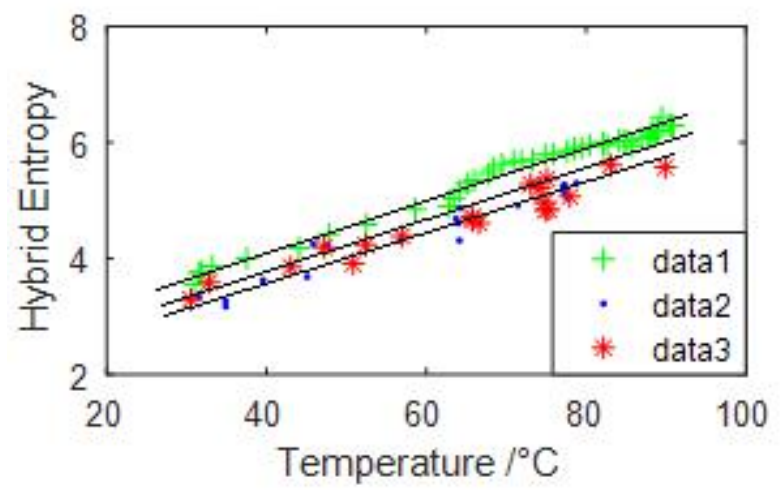

Fig.5 Correlation between hybrid entropy and temperature in multiple sets of data in vitro

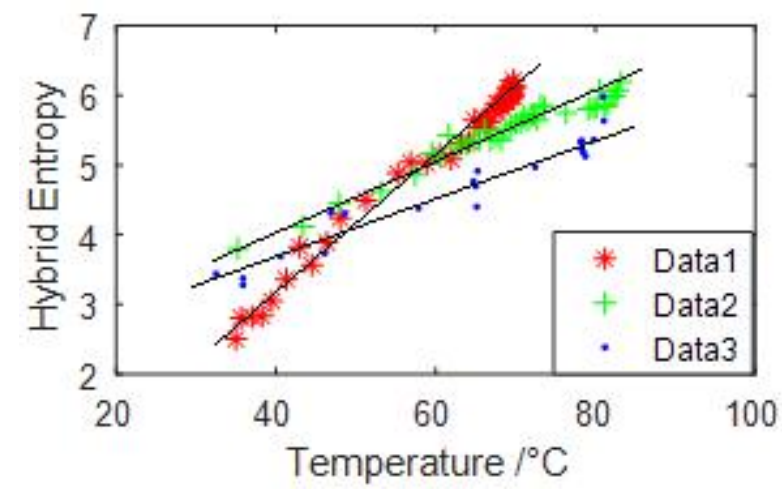

Fig.6 Correlation between hybrid entropy and temperature in multiple sets of data in vitro

It is speculated that the factors of slope difference between groups in vivo mainly include the following two points: (1) Difference in the ablation speed. Although the measured temperature was constantly rising in all groups, the duration of ablation at the same temperature was not the same. Therefore, image texture feature corresponding to the same temperature in different groups could be very different which had a certain impact on the linear relationship of feature and temperature in 
144 different groups in vivo. (2) Difference in biological materials. The biological tissue 145 characteristics of different rabbits for experiment were varied widely, so the 146 individual differences could be one of the most important factors which affect the 147 consistency of multiple sets of data.

\section{Conclusion}

This paper proposed an noninvasive temperature estimation method based on B-mode ultrasound image using wavelet transform and gray-level gradient co-occurrence matrix. It explored the relationship between texture features of ultrasound image and temperature both in vitro and in vivo. The radio frequency ablation experiments were ablation rate and the individual differences in tissue characteristics of biomaterials. performed on two kinds of biological material(pig kidney in vitro and rabbit kidney in vivo), during which the ultrasound image and temperature data were collected. After data acquisition, wavelet transform was performed on ultrasound subtraction image to enhance the detailed texture, and features of the gray gradient-level co-occurrence matrix that have a high correlation with temperature were extracted from processed image.

It is showed that under the two different experimental conditions, hybrid entropy and energy extracted with this method have an obvious linear relationship with temperature. The slopes of linear regression model obtained in vitro are relatively consistent, while those under in vivo condition have certain differences affected by The result under in vivo condition verifies the feasibility of the noninvasive 
temperature estimation method proposed in practice. However, the process of in vivo experiment still has some shortcomings.

Although the rabbits in experiment were anesthetized, it was impossible to avoid the influence of breathing and other tiny movements on the positioning of the area of interest. In this paper, the manual positioning method we used is more time-consuming, so some automatic positioning methods will be designed to achieve high efficiency and accuracy in segmentation in future work . In addition, although the models obtained from multiple groups of in vivo experiments have high linear correlation, there is still some difference in slope. Therefore, how to eliminate the differences and establish a general prediction model is still the focus of exploration in the future research.

\section{Methods}

\section{Experimental system}

The experimental system consists of radio frequency ablation system, temperature measurement system, B-mode ultrasound machine and biological materials, as shown in Fig.7. The experimental platform is shown in Fig.8. The radio frequency ablation system Cool-tip from Valleylab is mainly composed of radio frequency generator with an adjustable power in the range of $0 \mathrm{~W} \sim 200 \mathrm{~W}$, a radio frequency electrode and condensing circulatory system. The radio frequency ablation in experiment is guided by B-ultrasound machine Flex Focus 800 from BK medical company with convex array 8233 ultrasonic probe. The temperature measurement system is based on 
NI9213 thermocouple temperature measurement module with a conversion time of $55 \mathrm{~ms}$ and a channel sampling rate of 1 sample/s, the temperature data which collected can be displayed and stored in real time on the computer.

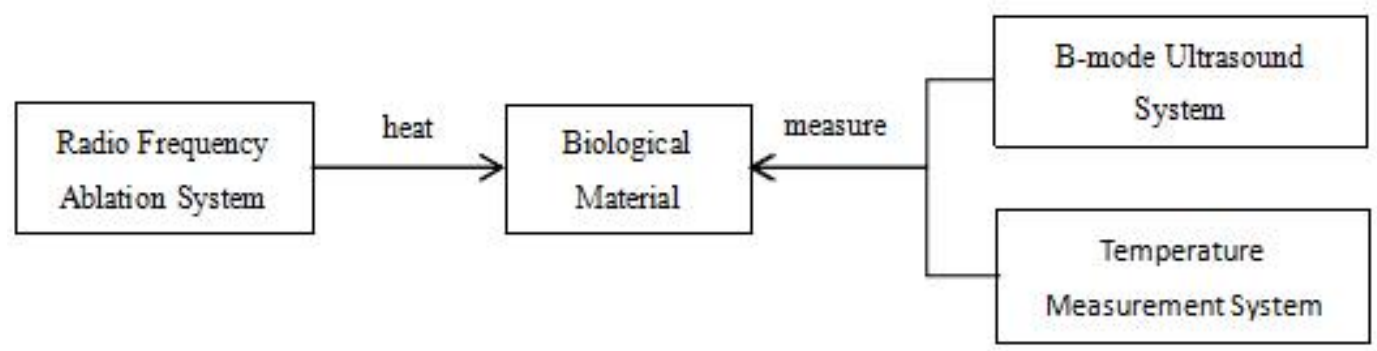

Fig.7 Experiment scheme

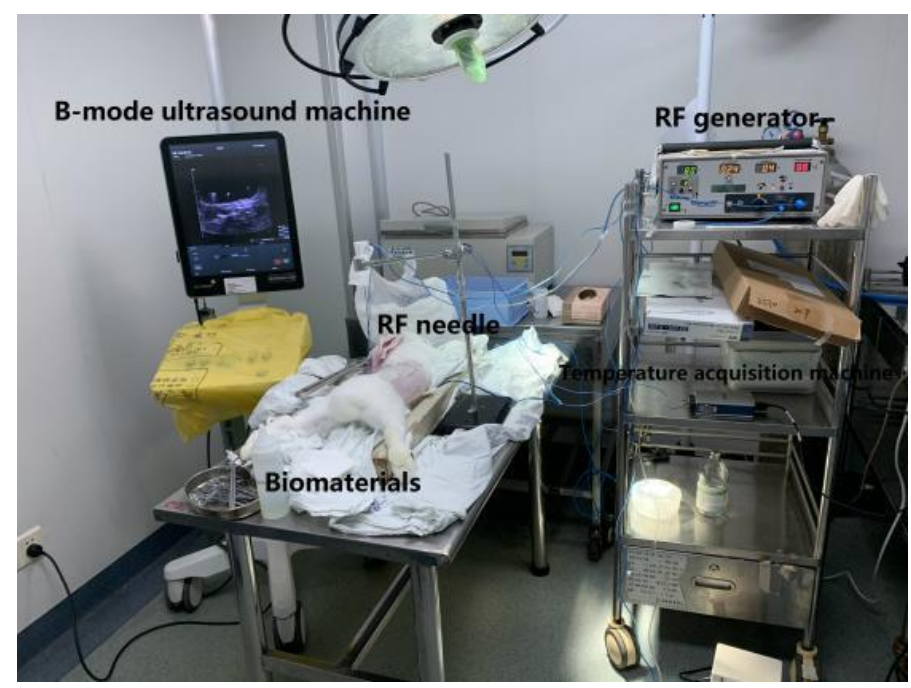

Fig.8 Experiment platform

As for experimental materials, pig kidney tissue and rabbit kidney tissue are both similar to human kidney tissue in composition. For in vitro experiment, due to larger size and contact area with the thermal ablation plate which were conducive to the experiment, pig kidney was chosen as experimental material. For in vivo experiment, rabbit kidney in vivo was used as the biological material because breathing and other motions of rabbit under anesthesia were smaller so that there would be fewer factors unrelated to the experiment. 


\section{Experimental method}

The experiment was conducted according to the following two steps. (1) Pretreatment of biological materials: The rabbit was anesthetized with its kidney exposed as ablation experiment material by surgery and abdomen dehaired to ensure its good contact with the electrode plate. The pig kidney in vitro was placed horizontally on the electrode plate to ensure good contact with the electrode plate. After placing the biological materials, the radio frequency ablation probe and temperature probe should be inserted vertically into the kidney with a depth of about $1 \mathrm{~cm}$ so that the two probe tips would be on the same horizontal line. After the probes were placed, B-ultrasound probe should be placed close to the surface of the kidney and perpendicular to the the other two probes and ensured that its center height would be the same as the height of the other two probe tip. (2) Radio frequency ablation and data acquisition: Based on previous experience ${ }^{[11]}$, the duration of ablation was set to $8 \mathrm{~min}$, and the ablation power was adjusted manually during the experiment to make sure the temperature change steady. Radio frequency ablation system, ultrasound image system and temperature measurement system were turned on at the same time, and 2 minutes after ablation finished, image recording of the B-mode ultrasound machine and temperature measurement system were stopped simultaneously.

\section{Image preprocessing}

The video frame rate of the ultrasound image obtained is 22 frames/s. In order to eliminate random noise and make the sampling frequency of ultrasound image and temperature data the same, we calculated the average value of each pixel of the 

shown in Fig.9.
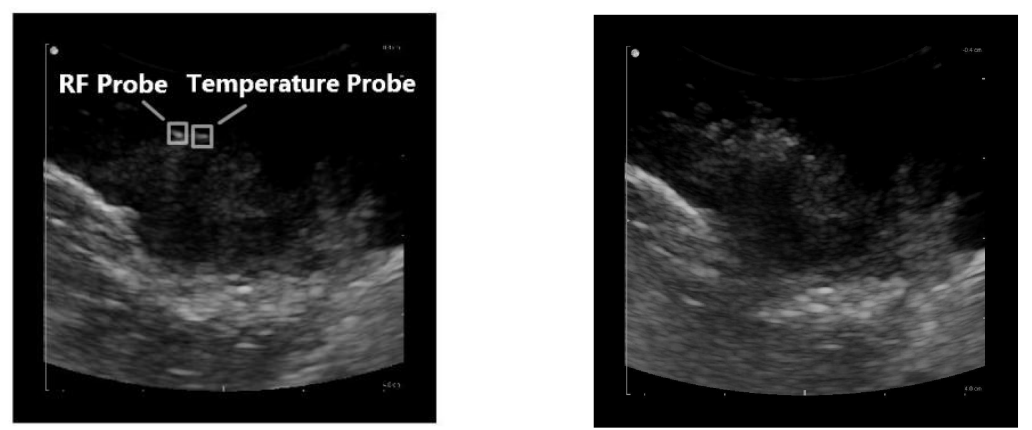

226 (a) Ultrasound image before ablation experiment (b) Ultrasound image after ablation experiment

Fig.9 Ultrasound images before and after the ablation experiment

A 40-pixel $\times 40$-pixel region centered on temperature probe was intercepted as the region of interest for processing which corresponding to the temperature obtained. In order to avoid the interference of the temperature probe and reduce the error under different conditions, the difference processing of the ultrasound images before and during RFA was performed, and then wavelet transform and texture analysis were performed on the subtraction image obtained.

\section{Wavelet transform}

Wavelet transform has the ability of multi-resolution analysis and characterizing local signals in both time and frequency domains. To perform a two-dimensional discrete wavelet transform on an image, Mallat algorithm can be used to achieve a pyramidal structure of image. After the image is decomposed by wavelet, it will produce two parts, low frequency component and high frequency component. The low frequency component reflects the approximate part of the image, and the high frequency 
component carries the information of the texture details ${ }^{[12]}$.

Because we pay more attention to the change of ultrasound image during ablation experiment, the detail components of the wavelet decomposition of the subtraction image were used for wavelet reconstruction to highlight the texture details of the ultrasound image, which provided a basis for the next step of using the gray-level gradient co-occurrence matrix to analyze image texture features.

\section{Gray-Level Gradient Co-occurrence Matrix}

Gray-Level Gradient Co-occurrence Matrix (GLGCM) is a method for image texture feature analysis, which reflects not only the gray spatial distribution but also edge gradient information. Gray spatial distribution information is the basis of the image composition, and gradient information constitutes the edge of the image. The combination of the two key information can better describe texture characteristics of a image ${ }^{[13]}$.

The operational processes of how to extract the gray-gradient co-occurrence matrix from a image are as follows. $f(i, j)$ is the gray image to be processed, from which we can obtain gradient image $\mathrm{g}(i, j)$ by using gradient operator. The gray matrix $F(i, j)$ and gradient matrix $G(i, j)$ can be obtained from these images by regularization processing shown in Eq.1 and Eq.2, where $L$ and $L_{g}$ are number of gray levels of gray image and gradient image respectively, $f_{\max }$ and $g_{\max }$ are the max value of gray image and gradient image respectively.

$$
F(i, j)=\frac{f(i, j) L}{f_{\max }}+1
$$




$$
G(i, j)=\frac{g(i, j) L_{g}}{g_{\max }}+1
$$

264 The gray gradient co-occurrence matrix $H(x, y)$ is donated as the number of the 265 pixels, which gray value is $\mathrm{x}$ in $F(i, j)$ and the gradient is $\mathrm{y}$ in $G(i, j)$. In order to simplify the calculation, the gray-level gradient co-occurrence matrix is normalized to make the sum of every element to be 1, as shown in Eq.3.

$$
H^{\prime}(i, j)=\frac{H(i, j)}{\sum_{i=0}^{L-1} \sum_{j=0}^{L_{g}-1} H(i, j)}
$$

Based on the normalized gray gradient co-occurrence matrix, 15 second-order feature parameters can usually be used to describe the texture characteristics of the image, including small gradient dominance, large gradient dominance, gray mean, gradient mean, gray entropy, gradient entropy, hybrid entropy, gray asymmetry, gradient asymmetry, energy, gray standard deviation, gradient standard deviation, correlation,

274 inertia and inverse difference moment. Based on the research characteristics, hybrid entropy and energy were selected as the characteristics to estimate temperature, as shown in Eq.4 and Eq.5.

Hybrid entropy:

$$
T_{1}=-\sum_{i=0}^{L-1} \sum_{j=0}^{L_{g}-1} H^{\prime}(i, j) \log _{2} H^{\prime}(i, j)
$$

279 Energy:

$$
T_{2}=\sum_{i=0}^{L-1} \sum_{j=0}^{L_{g}-1} H^{\prime 2}(i, j)
$$

\section{References}


[1] Shultz Kim,Stang Pascal,Kerr Adam,Pauly John,Scott Greig. RF field visualization of RF ablation at the Larmor frequency.[J]. IEEE transactions on medical imaging,2012,31(4).

[2] Giulio Di Candio MD, Francesco Porcelli MD, Alessandro Campatelli MD, et al. High-Intensity Focused Ultrasonography and Radiofrequency Ablation of Renal Cell Carcinoma Arisen in Transplanted Kidneys: Single-Center Experience With Long-Term Follow-Up and Review of Literature. 2019, 38(9):2507-2513.

[3] Hildebrandt B, Wust P, Ahlers O, et al.The cellular and molecular basis of hyperthermia[J].Crit Rev Oncol Hema.tol, 2002, 43 (1) :33.56.

[4] Hue Yik-Kiong,Guimaraes Alexander R,Cohen Ouri,Nevo Erez,Roth Abraham,Ackerman Jerome L. Magnetic Resonance Mediated Radiofrequency Ablation.[J]. IEEE transactions on medical imaging,2018,37(2).

[5] Jenista, Branca, Warren. Absolute temperature imaging using intermolecular multiple quantum MRI. International Journal of Hyperthermia, 2010, 26(7):725-734.

[6] H.Woo,Y. Kim,A.Guy. Feasibility Study of Monitoring Temperature Rise in Muscle Phantoms by the Electrical Impedance Tomography System During Hyperthermia Treatment[J]. Journal of Microwave Power and Electromagnetic Energy,2016,25(4).

[7] Hou Z X, et al.A new experimental study on noninvasive thermometry in HIFU[J].Journal of Harbin Institute of Technology,2002(03):259-262.

[8] Yang Chunlan, Zhu Hao, Wu Shuicai, et al. Correlations between B-mode ultrasonic image texture features and tissue temperature in microwave ablation.. 2010, 29(12):1787-99.

[9] LI Yong, DING Yajun, QIAN Shengyou, et al. Noninvasive temperature estimation method based on gray probability distribution-cross entropy[J]. Journal of Electronic Measurement and 
Instrumentation,2015,29(02):247-251.

[10] GUO Yan, DING Yajun, QIAN Shengyou, CHEN Xing. Noninvasive Temperature Measurement Based on BEMD and Random Forest Algorithm in High Intensity Focus Ultrasound Therapy[J]. Journal of Test and Measurement of Technology,2018,32(06):487-492.

[11] CUI Mengyao, YING Xiaoting, ZHAO Xingqun, YAO Linfang. Correlation between renal ultrasound image features and temperature during radio frequency ablation. Chinese Journal of Medical Physics, 2020, 37(9): 1164-1168.

[12] Kim Yong Sun,Ra Jong Beom. Speckle Noise Reduction and Edge Enhancement in Ultrasound Images Based on Wavelet Transform $[\mathrm{J}]$. Journal of Biomedical Engineering Research,2008,29(2).

[13] Lam SW-C. Texture feature extraction using gray level gradient based co-occurence matrices. 1996 IEEE International Conference on Systems, Man and Cybernetics Information Intelligence and Systems (Cat No96CH35929). 1996;1:267.

\section{Declaration}

\section{Ethics approval and consent to participate}

The experiment was approved by the ethics committee of Nanjing Drum Tower Hospital, the Affiliated Hospital of Nanjing University Medical School, Nanjing, China. Animals were managed according to the guidelines of the American Physiological Society.

\section{Consent for publication}

Not applicable. 
The datasets during and/or analysed during the current study available from the

329 corresponding author on reasonable request.

\section{Competing interests}

331 The authors declare that they have no competing interests.

\section{$332 \quad$ Funding}

333 None declared.

\section{Author information}

\section{Affiliations}

336 School of Biological Science and Medical Engineering, Southeast University, Nanjing,

337 China

338 Ming Chen \& Xingqun Zhao

339 Department of Urology, Nanjing Drum Tower Hospital, the Affiliated Hospital of

340 Nanjing University Medical School, Nanjing, China

341 Linfang Yao

\section{$342 \quad$ Authors' contributions}

343 MC contributed to data collection, processing, analysis and manuscript drafting. LY

344 and $\mathrm{MC}$ contributed to design and operation of the experiment. $\mathrm{XZ}$ and LY

345 contributed to the manuscript revision. All authors approve the final version of the

346 manuscript.

\section{Corresponding author}

348 Correspondence to Xingqun Zhao \& Linfang Yao. 


\section{Acknowledgements}

$350 \quad$ Not applicable. 
Figures

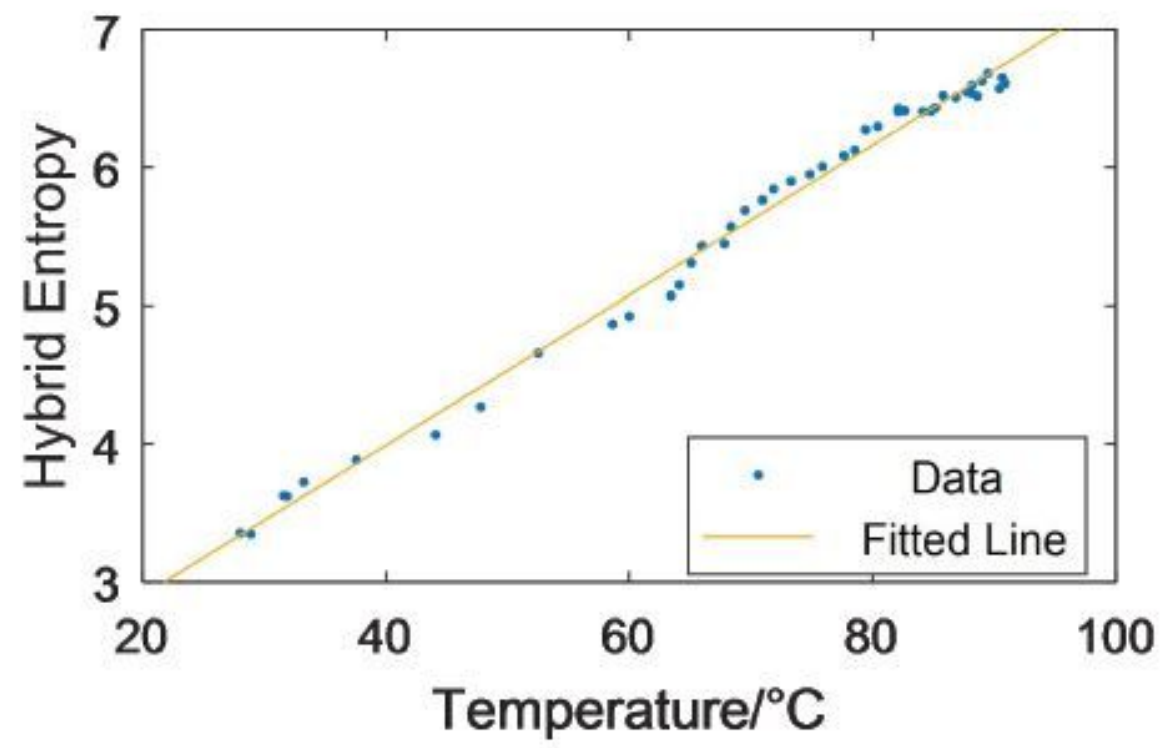

(a) Correlation between hybrid entropy and temperature

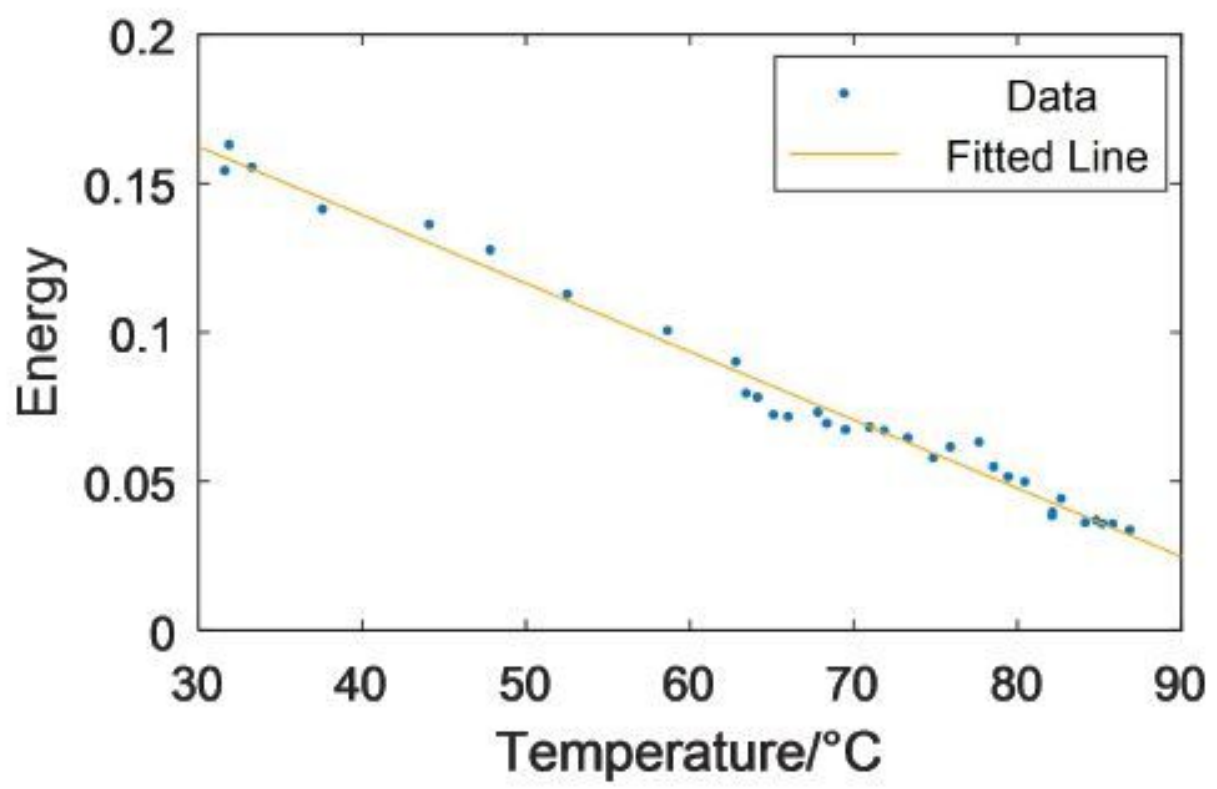

(b) Correlation between energy and temperature

Figure 1

Correlation between texture features and temperature in vitro experiment 


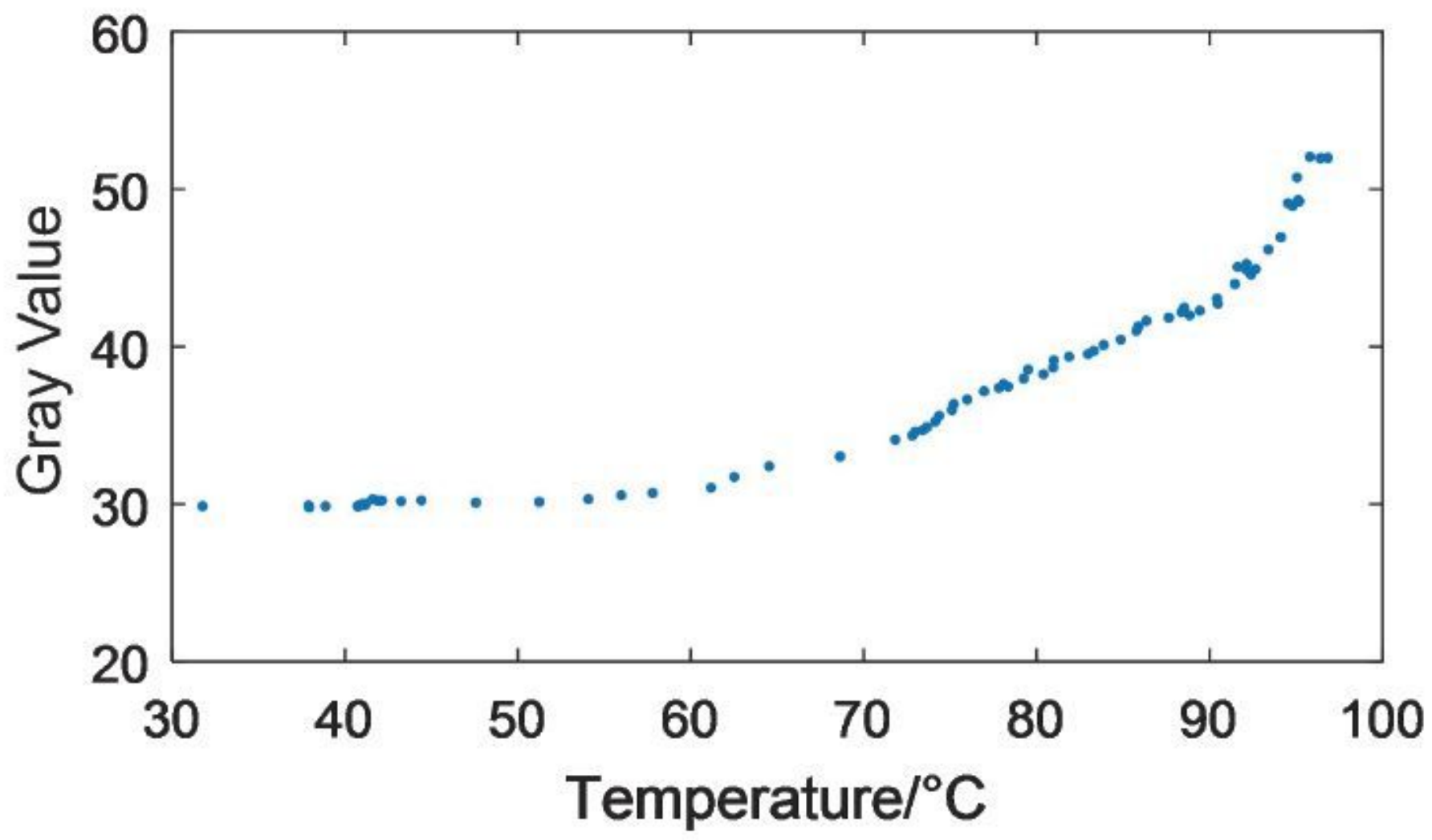

Figure 2

Correlation between gray value and temperature in vitro experiment 


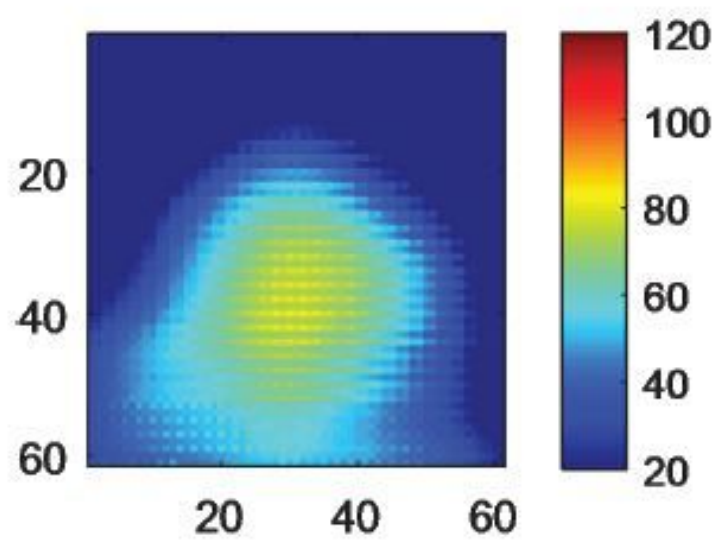

(a) Time $=100 \mathrm{~s}$

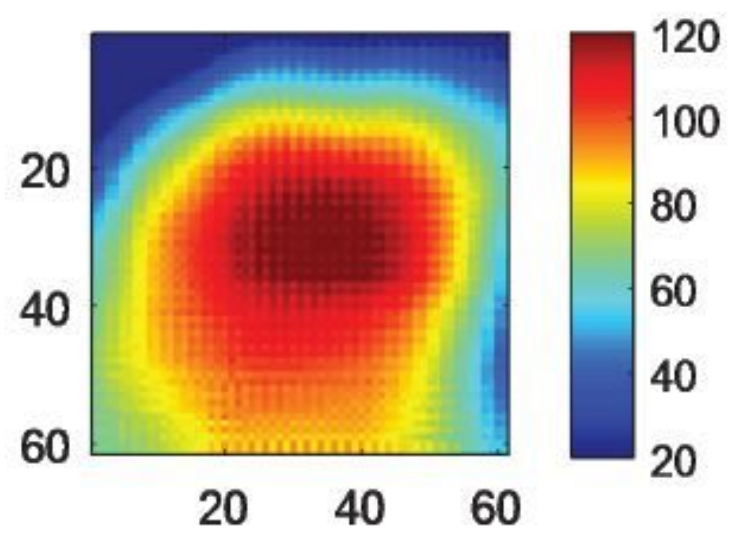

(c) Time $=300 \mathrm{~s}$

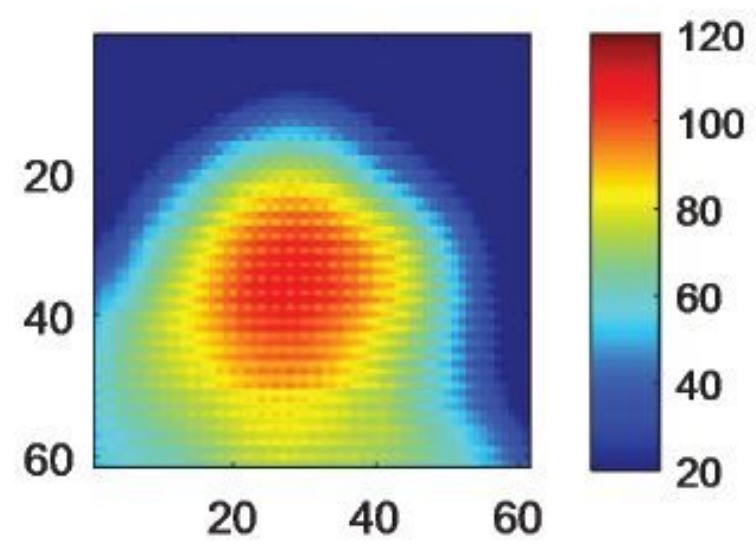

(b) Time $=200 \mathrm{~s}$

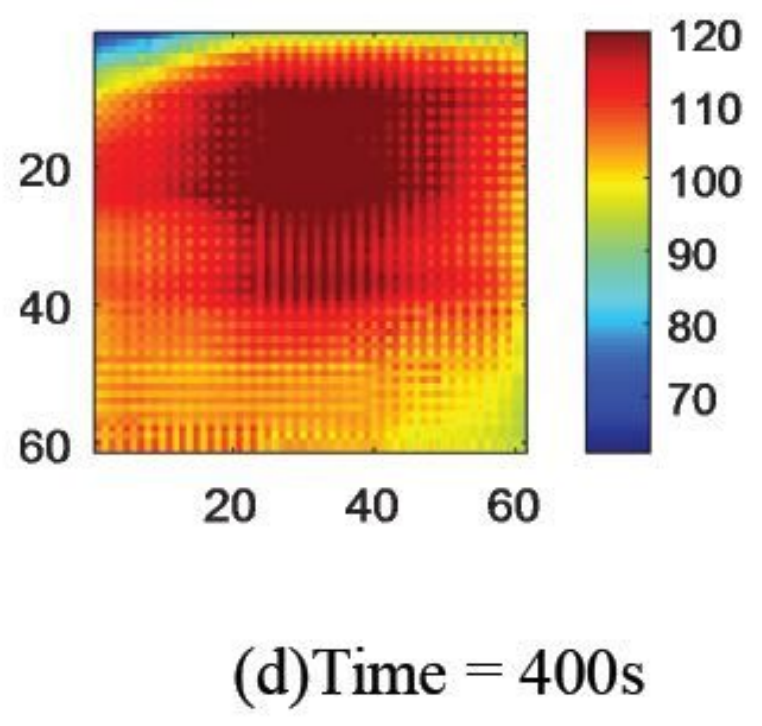

Figure 3

Pseudo-color image of temperature distribution 


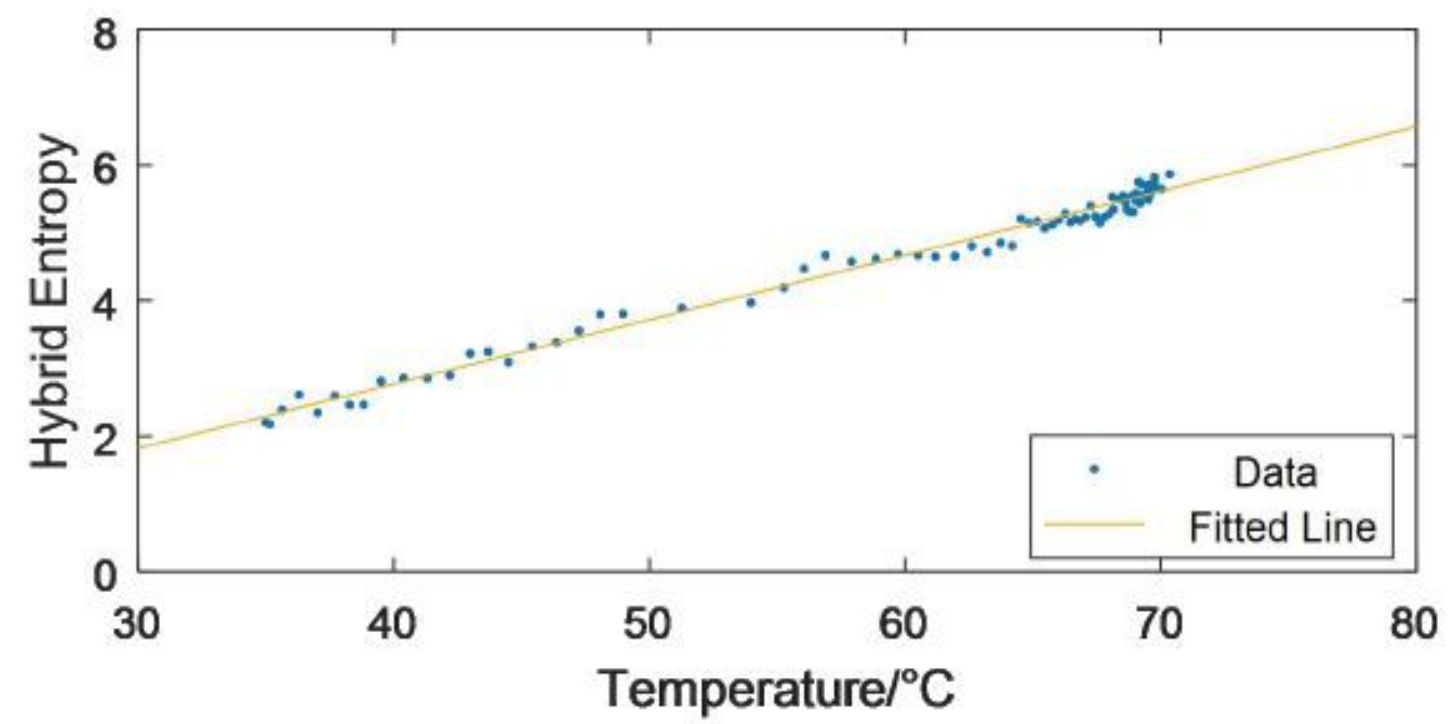

(a) Correlation between hybrid entropy and temperature

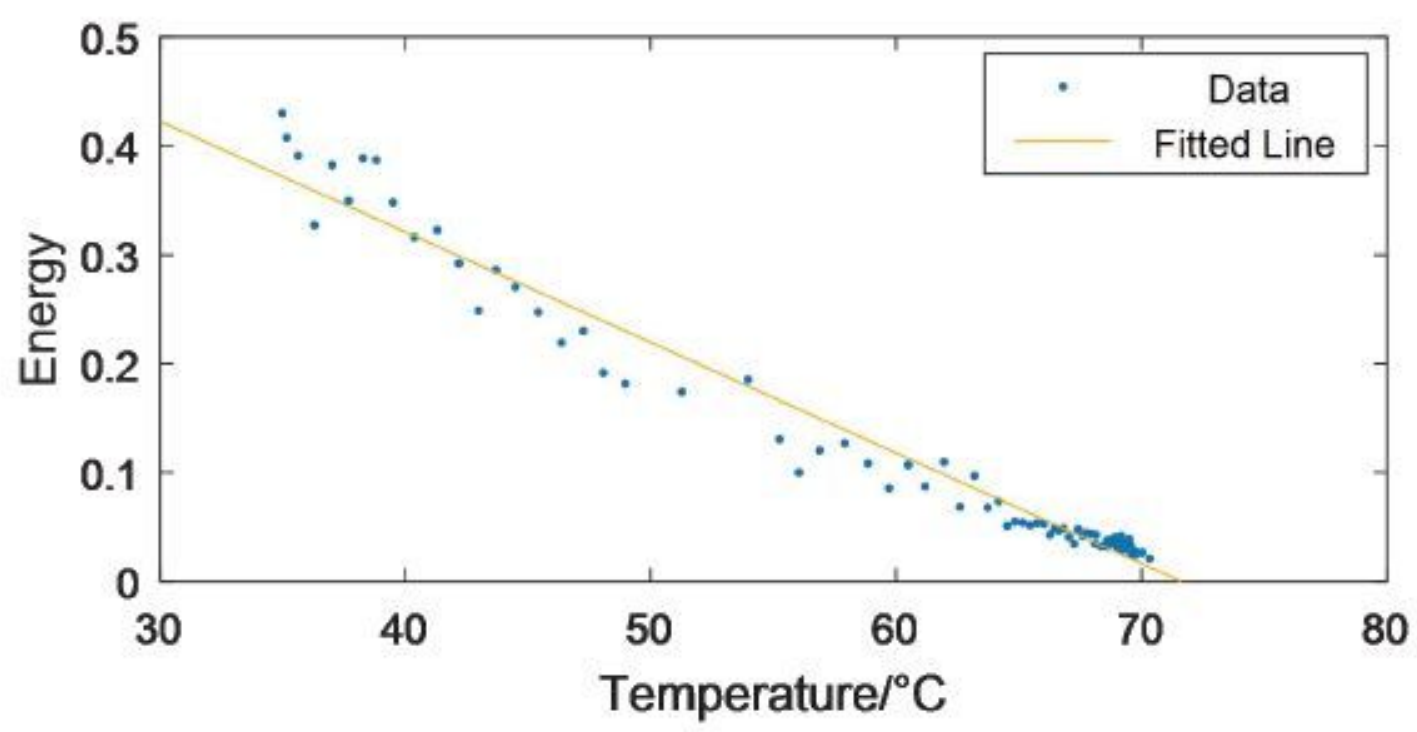

(b) Correlation between energy and temperature

Figure 4

Correlation between features and temperature from in vitro experiment 


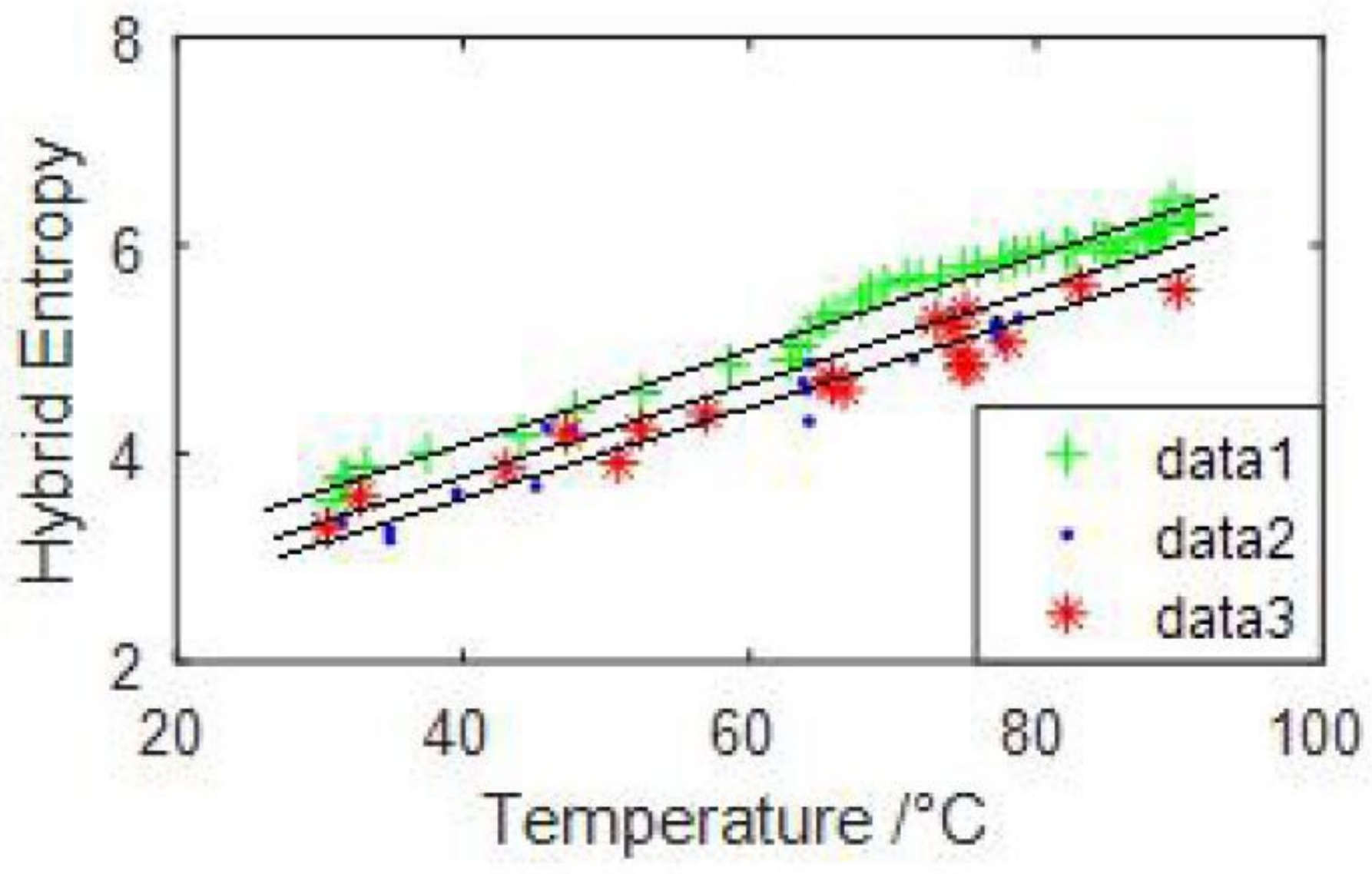

Figure 5

Correlation between hybrid entropy and temperature in multiple sets of data in vitro 


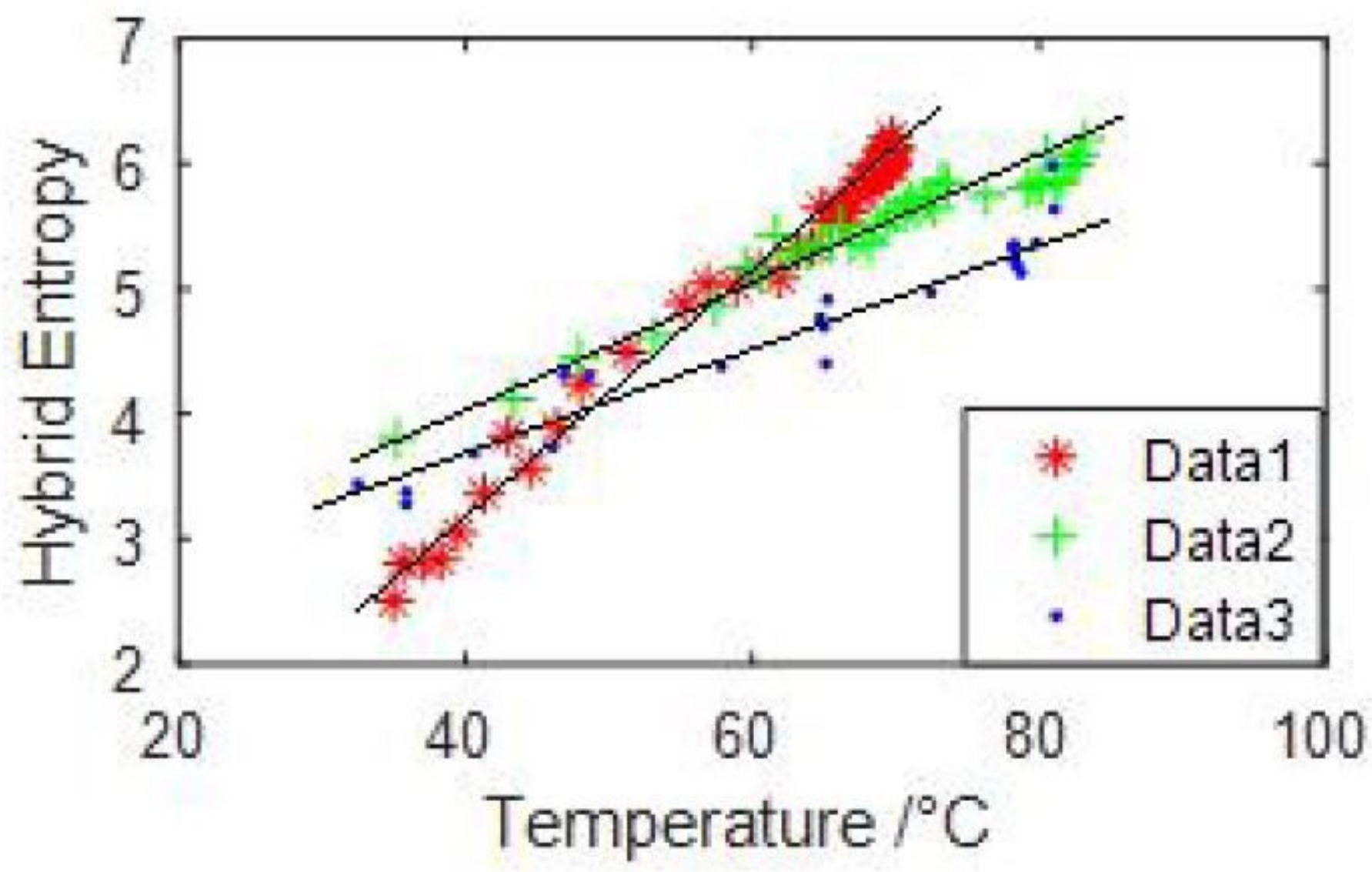

Figure 6

Correlation between hybrid entropy and temperature in multiple sets of data in vitro

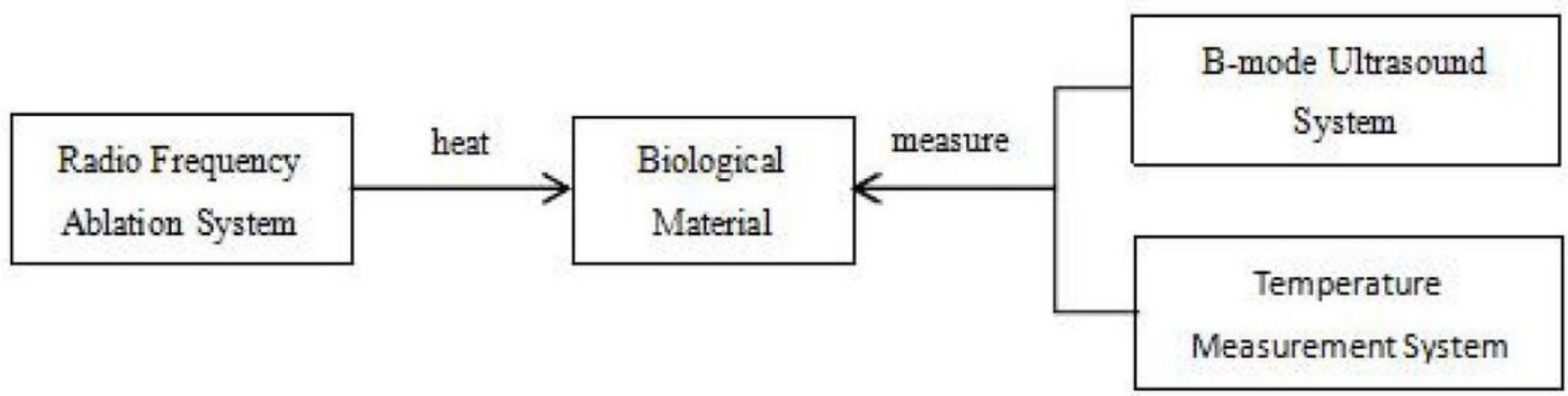

Figure 7

Experiment scheme 


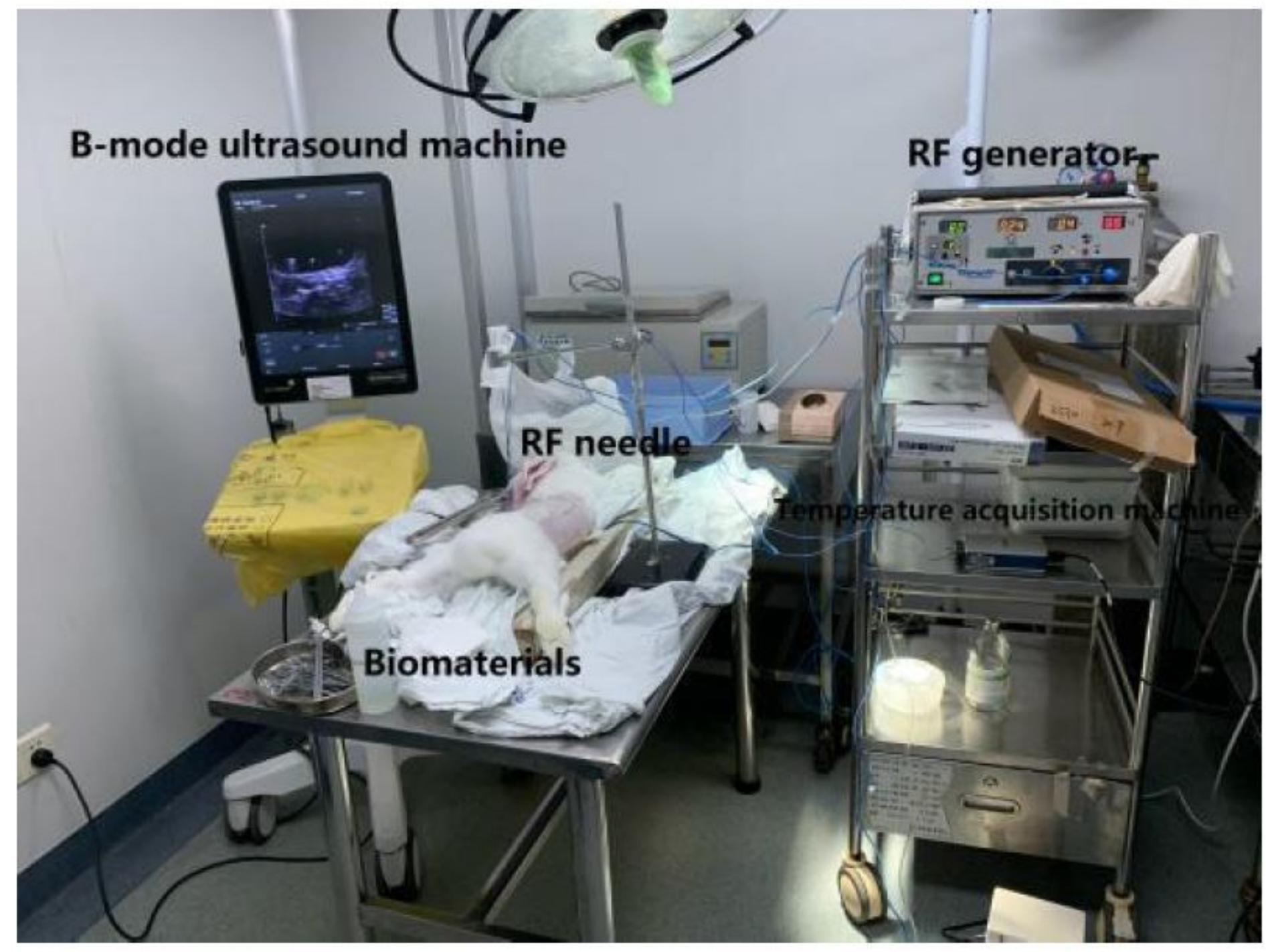

Figure 8

Experiment platform 


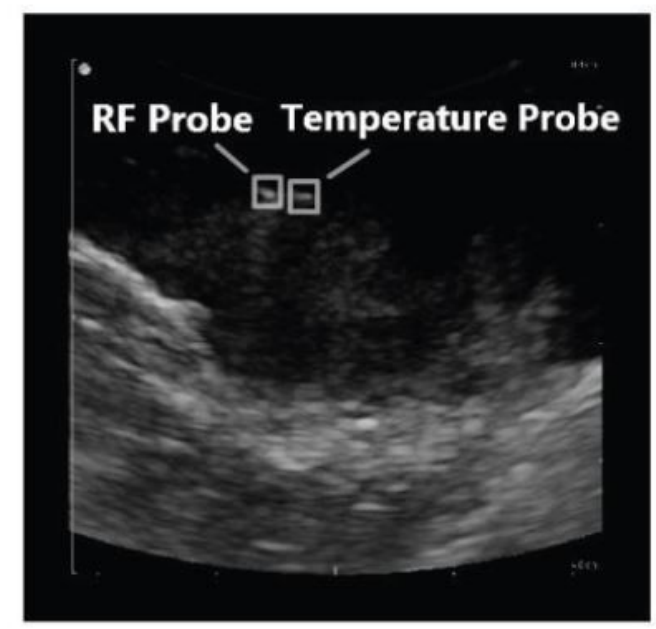

(a) Ultrasound image before ablation experiment

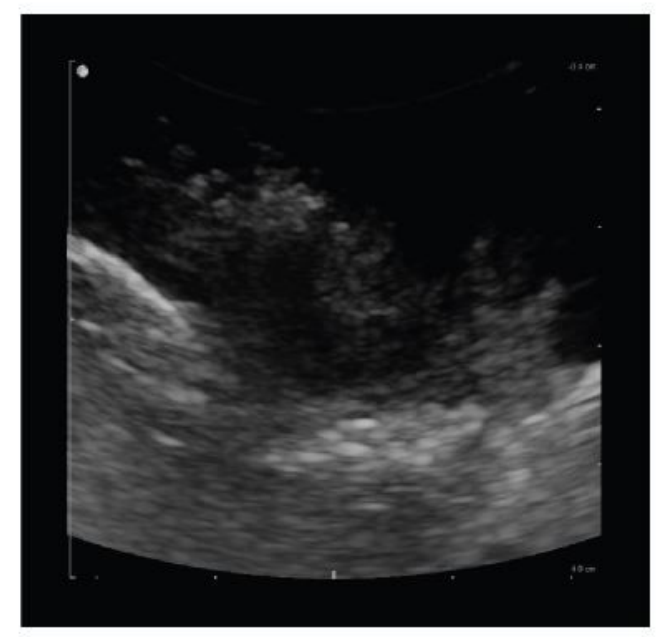

(b) Ultrasound image after ablation experiment

\section{Figure 9}

Ultrasound images before and after the ablation experiment. (a) Ultrasound image before ablation experiment (b) Ultrasound image after ablation experiment 